\title{
Doppler Frequency Estimation for a Maneuvering Target Being Tracked by Passive Radar Using Particle Filter
}

\author{
Anas Mahmoud Almanofi, Adnan Malki, and Ali Kazem
}

\begin{abstract}
In this paper, we estimate Doppler frequency of a maneuvering target being tracked by passive radar using two types of particle filter, the first is "Maximum Likelihood Particle Filter" (MLPF) and the second is "Minimum Variance Particle Filter" (MVPF). By simulating the passive radar system that has the bistatic geometry "Digital Video Broadcasting-Terrestrial (DVB-T) transmitter / receiver" with these two types, we can estimate the Doppler frequency of the maneuvering target and compare the simulation results for deciding which type gives better performance.
\end{abstract}

Index terms - Passive Radar, Doppler Frequency, Maneuvering Target, Maximum Likelihood Particle Filter, Minimum Variance Particle Filter.

\section{INTRODUCTION}

$\mathrm{P}$ ASSIVE radar is a bistatic radar that detects and tracks targets by processing reflections from non-cooperative sources of illumination. It has many advantages, such as resistant to jammers and does not need a dedicated frequency band. This radar is equipped with two antennas: The first antenna is the direct antenna that receives the direct signal, and the second antenna is the surveillance antenna that receives targets' echoes and multipath signals [1], [2]. The direct signal is reconstructed to detect targets' echoes [3]-[5]. Many researches have been conducted studying this radar, such as studying and analyzing of signals of non-cooperative transmitters (e.g., Frequency Modulation (FM) radio, Global System for Mobile communication (GSM), Digital Video Broadcasting-Terrestrial (DVB-T), and Digital Audio Broadcasting (DAB)) [1], [2], [6]-[9], studying of the interference of the direct signal on the surveillance channel [10], [11], detection of "maneuvering/non-maneuvering" targets [12]-[14], and estimation of their parameters (e.g.,

Manuscript received June 20, 2020; revised September 7, 2020. Date of publication October 8, 2020. Date of current version October 8, 2020. The associate editor prof. Zoran Blažević has been coordinating the review of this manuscript and approved it for publication.

Authors are with the Department of Communication Engineering, Higher Institute for Applied Sciences and Technology (HIAST), Damascus, Syria. (e-mails: \{anas.almanofi, adnan.malki, ali.kazem\} @hiast.edu.sy).

Digital Object Identifier (DOI): 10.24138/jcomss.v16i4.1097
Doppler frequency, velocity, acceleration, and coordinates) [2], [15]-[19].

Doppler frequency of a non-maneuvering target is estimated by applying the Maximum Likelihood method to the output of a bank of matched filters, which are tuned to different Doppler frequencies [1], [17]. But in the case of a maneuvering target, this way is ineffective because the velocity and direction of this target change non-linearly during small periods. Therefore, non-linear tracking filters should be used, such as Extended Kalman Filter (EKF), Unscented Kalman Filter (UKF) [20]-[22], and Particle Filter (PF) [22]-[31]. Many researches have indicated that the $(\mathrm{PF})$ has better performance for estimating parameters that change non-linearly at low SNR, because it depends on the Monte Carlo method, [2], [22]-[24]. According to the processing method, the (PF) has the following two types: MLPF and MVPF [23], [24]. In this paper, we estimate the Doppler frequency of a maneuvering target by simulating these two types within a passive radar system that has the bistatic geometry "DVB-T transmitter/receiver". Then we suggest the optimal application for each type by comparing and analyzing the simulation results.

The paper is organized as follows. Section II introduces the passive radar system with a maneuvering target. Section III illustrates the particle filter with its two types (MLPF and MVPF), which will be used for estimating the Doppler frequency for the maneuvering target. Section IV shows simulating the passive radar system with these two types and concentrates on discussing the simulation results. Section V concludes the paper.

\section{PASSIVE RADAR SYSTEM}

It has the following bistatic geometry: one DVB-T transmitter and one receiver with two receiving antennas, taking into consideration that there is one maneuvering target, as shown in Fig. 1 where $T_{x}$ is the non-cooperative transmitter, $R_{x}$ is the receiver, $T_{a}$ is the maneuvering target, $R_{1}$ is the range between the transmitter $\left(T_{x}\right)$ and the target $\left(T_{a}\right), R_{2}$ is the 


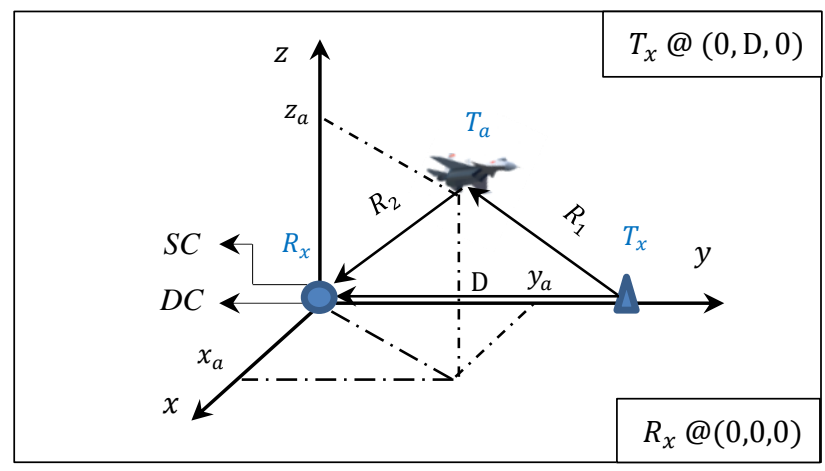

Fig. 1. Bistatic geometry of passive radar

effective range, $\left(x_{a}, y_{a}, z_{a}\right)$ are the Cartesian coordinates of the observed target $\left(T_{a}\right), \mathrm{D}$ is the distance from the transmitter $\left(T_{x}\right)$ to the receiver $\left(R_{x}\right), S C$ refers to "Surveillance Channel", and $D C$ refers to "Direct Channel".

After suppressing the interference in the surveillance channel and mitigating multipath signals based on the properties of the DVB-T signal that uses the Orthogonal Frequency Division Multiplexing (OFDM) modulation technique, the echo signal of the observed target is given in (1), [2], [23], [25].

$$
Y(t)=A(t) e^{j \emptyset(t)} S(t-\tau)+n(t) ; \quad t=0: T_{p}
$$

where $t$ is the discrete time of the observation, $Y$ is the observation signal, $A$ is the amplitude, $\emptyset$ is the phase, $S$ is the delayed direct signal with the time delay $(\tau), n$ is the Additive White Gaussian Noise, and $T_{p}$ is the duration of the processed data window.

For estimating the Doppler frequency of the maneuvering target by the particle filter, we will briefly explain this filter and its two types, as presented in the following section.

\section{PARTICLE FILTER}

It is a filtering method that depends on the Monte Carlo approximation and recursive Bayesian estimation. It mainly consists of propagating, in a non-linear way, weighted particles in a domain of a studied state. With helping of observations from a studied system, the weight and state of each particle are processed by a method for getting estimation results. For effective estimation, a resampling step should be applied for re-propagating the particles in a different domain for the studied state [26], [27]. This process is achieved by processing the output of the following two equations for each particle: The state equation and measurement equation, which are given in (2) and (3), respectively [26]-[28].

$$
\begin{aligned}
& x_{t}=f\left(x_{t-1}\right)+v_{t} \\
& Z_{t}=h\left(x_{t}\right)
\end{aligned}
$$

where $(t,(t-1))$ are the current and previous measurement time, respectively, $x$ is the state vector $\left(x \in \mathbb{R}^{n_{x}}\right), f$ is a non- linear function and it is a known function, $v$ is the state noise vector $\left(v \in \mathbb{R}^{n_{v}}\right) ; v \sim \mathcal{N}\left(0, \sigma_{v}^{2}\right), Z$ is the measurement signal $\left(Z \in \mathbb{R}^{n_{Z}}\right)$, and $h$ is a non-linear function and it is a known function. The symbol $\left(\mathcal{N}\left(m, \sigma^{2}\right)\right)$ denotes the Gaussian density function with the mean $(m)$ and variance $\left(\sigma^{2}\right)$.

In the passive radar system, the state vector is $\left(x=\left[A, \emptyset, f_{d}, \tau\right]^{\mathrm{T}}\right)$, and the dynamics of its parameters are given in (4), [2], [23], [25].

$$
\left[\begin{array}{c}
A_{t} \\
\emptyset_{t} \\
f_{d_{t}} \\
\tau_{t}
\end{array}\right]=\left[\begin{array}{c}
A_{t-1} \\
\emptyset_{t-1}+2 \pi f_{d_{t-1}} T_{p} \\
f_{d_{t-1}} \\
\tau_{t-1}-\frac{f_{d t-1}}{f_{0}} T_{p}
\end{array}\right]+\left[\begin{array}{c}
v_{t}^{A} \\
v_{t}^{\emptyset} \\
v_{t}^{f_{d}} \\
v_{t}^{\tau}
\end{array}\right]
$$

where $f_{d}$ is the Doppler frequency, $\left(v^{A}, v^{\phi}, v^{f_{d}}, v^{\tau}\right)$ are the Gaussian noises, $f_{0}$ is the carrier frequency, and $\mathrm{T}$ denotes the transposition.

The particle filter has two types according to its processing method, as presented in the following two subsections.

\section{A. $M L P F$}

It depends on moving the particles' distribution toward the region of highest likelihood by using the (EKF) [29], [30]. It is achieved by implementing the following steps, taking into consideration that $(p)$ is the Probability Density Function [25], [26], [28], [31].

a) Initialization: Propagating particles with different states and equal weights. The initial weights are as follows: $\left(w_{t=0}^{i}=1 / N_{s}\right)$, where $N_{s}$ is the number of particles and $i$ is the index of these particles; $i=1: N_{s}$. See the red particles in Fig. 2.

b) Calculating the current weights of the particles at the time $(t)$ by the following equation, taking into consideration the green particles in Fig. 2.

$$
\begin{aligned}
w_{t}^{i} & =w_{(t-1)}^{i} * p\left(Y_{t} / x_{t}^{i}\right)=w_{(t-1)}^{i} * \mathcal{N}\left(Y_{t}-h\left(x_{t}^{i}\right), R_{t}\right) \\
& =w_{(t-1)}^{i} * \mathcal{N}\left(Y_{t}-Z_{t}^{i}, R_{t}\right)
\end{aligned}
$$

where $\left(w_{t}^{i}, w_{(t-1)}^{i}\right)$ are the current and previous weight for the particle $(i)$, respectively, and $R_{t}$ is the covariance matrix [25], [28], [31].

c) Normalizing the calculated weights by the following equation:

$$
w_{t}^{i}=w_{t}^{i} / \sum_{i=1}^{N_{s}} w_{t}^{i}
$$

d) The studied state is estimated by the following equation:

$$
\hat{x}_{t}=\sum_{i=1}^{N_{s}}\left(w_{t}^{i} * x_{t}^{i}\right)
$$


e) Resampling: The particles that have high weights are selected for propagating new particles with different states and equal weights; $\left\{w_{t}^{i}=\left(1 / N_{s}\right) ; i=1: N_{s}\right\}$. See the green and black particles in Fig. 2 .

f) Re-implementing the steps $(\mathrm{b} \rightarrow \mathrm{e})$ at each observation time $(t)$.

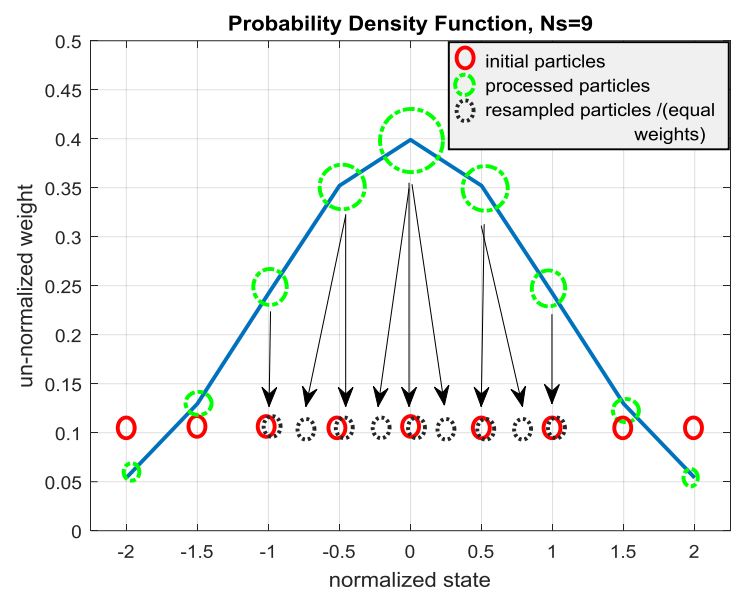

Fig. 2. Initial and resampled particles in case of MLPF

\section{B. $M V P F$}

It depends on analyzing the state probability of each particle by the Cumulative Distribution Function (CDF) [23], [24]. It is achieved by implementing the following steps [23], taking into consideration that $(\mathrm{F})$ denotes the CDF.

a) Initialization: Propagating particles with different states and equal weights; $\left(w_{t=0}^{i}=1 / N_{s}, i=1: N_{s}\right)$. See the red particles in Fig. 3.

b) Calculating the state probability of each particle at the time $(t)$ by the following equation:

$$
\mathrm{P}_{t_{\text {unnor }}}^{i}=\mathrm{F}\left(Y_{t}-h\left(x_{t}^{i}\right)\right)=\mathrm{F}\left(Y_{t}-Z_{t}^{i}\right)
$$

where $\mathrm{P}_{t_{\text {unnor }}}^{i}$ is the state probability of the particle $(i)$.

c) Resampling: The output of the CDF is sampled at the initial probability $\left(1 / 2 N_{s}\right)$ with the sampling resolution $\left(1 / N_{s}\right)$. The resulting states and probabilities are the states and un-normalized weights of the resampled particles, respectively. See the green particles in Fig. 3, which illustrates this process.

d) The studied state is estimated by the following equation:

$$
\hat{x}_{t}=\sum_{i=1}^{N_{s}}\left(\mathrm{P}_{t_{n o r}}^{i} * \ddot{x}_{t}^{i}\right)
$$

where $\left(\ddot{x}^{i}, \mathrm{P}_{t_{\text {nor }}}^{i}\right)$ are the state and normalized weight for the resampled particle $(i)$, respectively.

e) Updating the normalized weights of the resampled particles to $\left(1 / N_{s}\right)$. See the black particles in Fig. 3 . f) Re-implementing the steps $(b \rightarrow e)$ at each observation time $(t),[23]$.

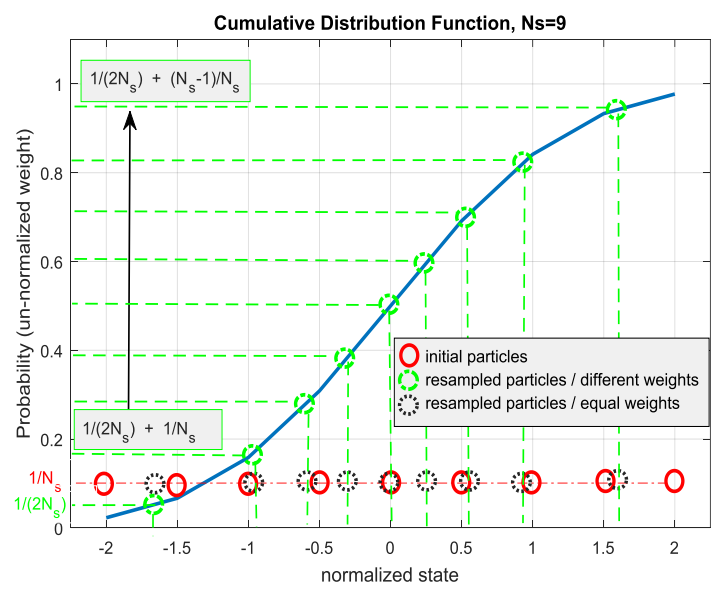

Fig. 3. Initial and resampled particles in case of MVPF

By focusing on Fig. 2 and Fig. 3, we notice that the variance between the resampled particles is equal in the case of MLPF and unequal in the case of MVPF. Therefore, MVPF has an additional advantage compared with MLPF, as presented later.

To illustrate the difference between MLPF and MVPF, we will suppose the following estimation with $N_{S}=$ 28 (particles). We want to estimate the real Doppler frequency that equals $(-781.8(\mathrm{~Hz}))$ by these two types, taking into consideration the proposed probability density for the weights of the processed particles, as shown in Fig. 4 and Fig. 5. By simulating the mentioned estimation, we notice that the resampled particles are distributed only around the real Doppler frequency (main event) in the case of MLPF, as shown in Fig. 4, whereas they are distributed around the main event and sudden event in the case of MVPF, as shown in Fig. 5. The sudden event cannot be observed in the case of MLPF, thus using (MVPF) will improve the performance of the passive radar for tracking targets in sudden environments.

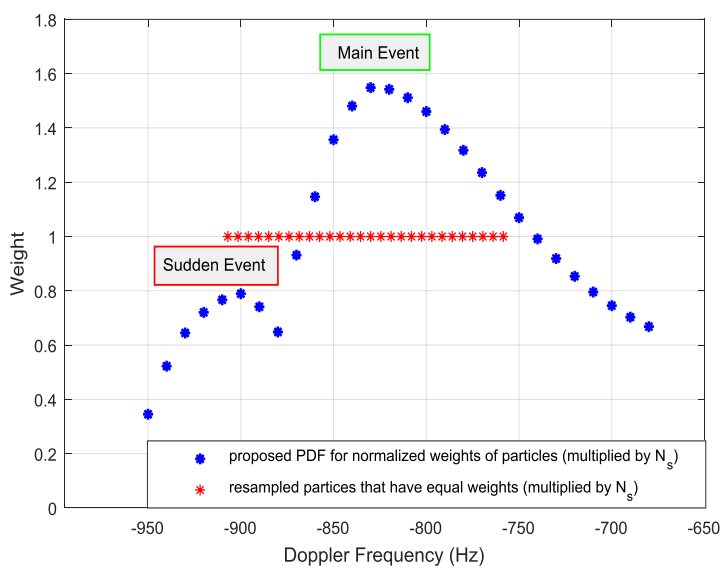

Fig. 4. Resampled particles in case of MLPF 


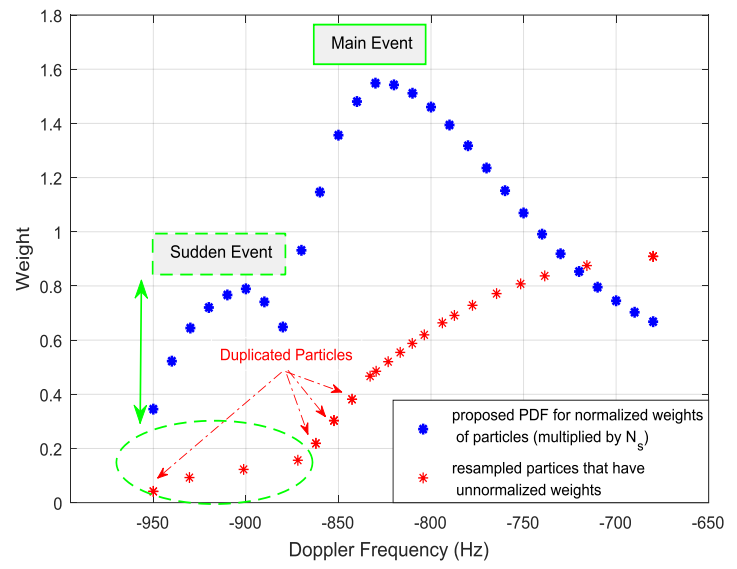

Fig. 5. Resampled particles in case of MVPF

\section{Simulation AND RESUlts}

\section{A. Simulation}

MATLAB software is used for simulating the particle filter with the passive radar system that consists of the DVB-T transmitter, Additive White Gaussian Noise channel with one maneuvering target, and receiver, as shown in Fig. 1. For simplicity, we consider that the parameters of the state vector $(A, \emptyset$ and $\tau)$ are determined at each measurement time.

This simulation is achieved with the technical characteristics for the transmitter, receiver and target, as listed in Table I.

TABLE I

TECHNICAL CHARACTERISTICS FOR TRANSMITTER, RECEIVER AND TARGET

\begin{tabular}{|c|c|c|c|c|}
\hline \multirow{5}{*}{ 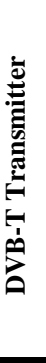 } & ERP & $50(\mathrm{KW})$ & Guard interval & $1 / 4$ \\
\hline & $\begin{array}{l}\text { Carrier } \\
\text { frequency }\end{array}$ & $\begin{array}{c}474 \\
(\mathrm{MHz})\end{array}$ & $\begin{array}{l}\text { Duration of } \\
\text { OFDM symbol }\end{array}$ & $1.1(\mathrm{~ms})$ \\
\hline & Bandwidth & $8(\mathrm{MHz})$ & $\begin{array}{l}\text { Cartesian } \\
\text { coordinates }\end{array}$ & $(0, D, 0)$ \\
\hline & $\begin{array}{l}\text { Transmission } \\
\text { mode/ } \\
\text { modulation }\end{array}$ & $\begin{array}{l}\text { 8K mode } \\
\text { / 64QAM }\end{array}$ & $\mathrm{D}$ & $5(\mathrm{Km})$ \\
\hline & Code Rate & $7 / 8$ & Losses $\left(L_{t}\right)$ & $1(\mathrm{~dB})$ \\
\hline \multirow{4}{*}{ 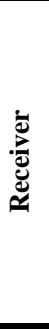 } & $\begin{array}{l}\text { Surveillance } \\
\text { antenna gain } \\
\left(G_{r_{S A}}\right)\end{array}$ & $22(\mathrm{~dB})$ & $\begin{array}{l}\text { Time difference } \\
\text { between two } \\
\text { consecutive } \\
\text { observations }\end{array}$ & $85(m s)$ \\
\hline & $\begin{array}{l}\text { Gain of direct } \\
\text { antenna }\end{array}$ & $2.5(\mathrm{~dB})$ & $\mathrm{T}_{\mathrm{P}}$ & $2.2(\mathrm{~ms})$ \\
\hline & $\begin{array}{l}\text { Cartesian } \\
\text { coordinates }\end{array}$ & $(0,0,0)$ & Losses $\left(L_{r}\right)$ & $1(\mathrm{~dB})$ \\
\hline & $\sigma_{v_{f_{d}}}^{2}$ & $1.8^{2}\left(H z^{2}\right)$ & Noise Figure $\left(F_{r}\right)$ & $2(\mathrm{~dB})$ \\
\hline 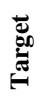 & $\begin{array}{l}\text { Monostatic } \\
\text { RCS }\end{array}$ & $6\left(m^{2}\right)$ & Initial coordinates & $\begin{array}{c}(9,8,3.5) \\
(K m)\end{array}$ \\
\hline
\end{tabular}

We consider that the observed target moves according to the path shown in Fig. 6, and its velocity changes according to Fig. 7. Therefore, the signal-to-noise ratio (SNR) of the target's echo signal changes according to Fig. 8, taking into consideration that the mentioned target is detected with a false alarm probability $\left(10^{-4}\right)$. The indicated parameter (SNR) is given in (10), [2], [7]

$$
S N R=\frac{P_{t} G_{t} G_{r_{S A}} \lambda^{2} \sigma_{R C S}}{(4 \pi)^{3} K T_{0} B F_{r} L_{t} L_{r} R_{1}^{2} R_{2}^{2}}
$$

where $P_{t}$ is the transmitted power (watt), $G_{t}$ is the transmitter antenna gain, $\lambda$ is the transmitter wavelength $(\mathrm{m}), \sigma_{R C S}$ is the bistatic radar cross section $\left(\mathrm{m}^{2}\right), K$ is Boltzmann's constant, $T_{0}$ is the effective noise temperature, and $B$ is the receiver bandwidth $(\mathrm{Hz})$.

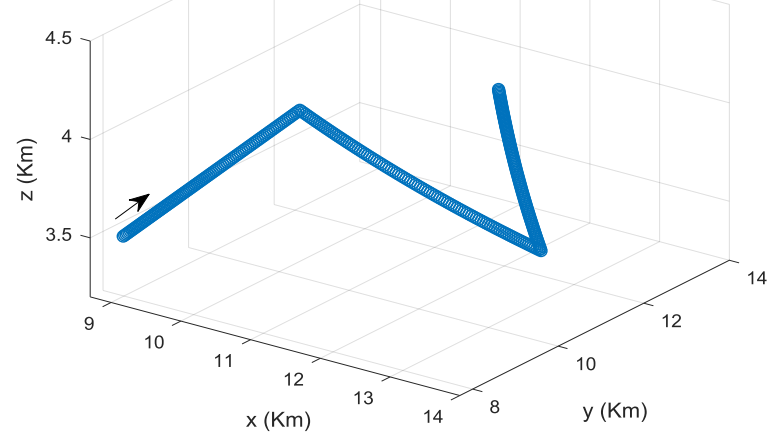

Fig. 6. Path of observed target

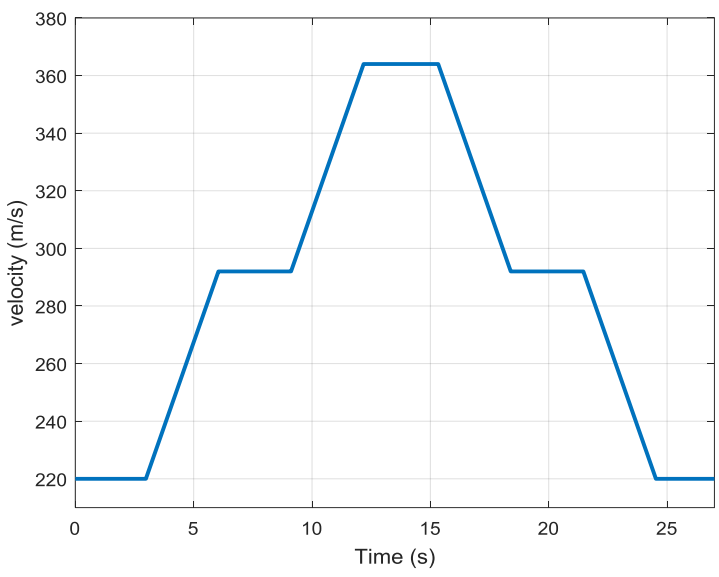

Fig. 7. Velocity of observed target as a function to time

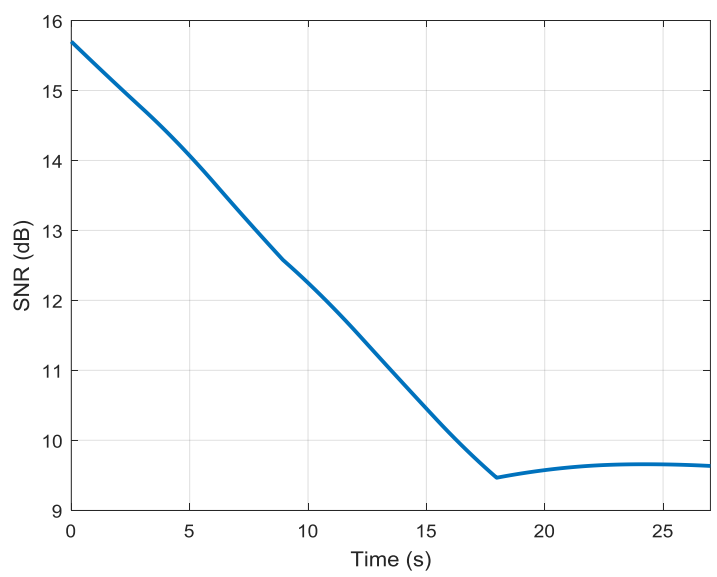

Fig. 8. SNR of target's echo signal as a function to time 


\section{B. Results and Notes}

We performed the estimation of the Doppler frequency of the maneuvering target by MLPF and MVPF for the following two cases: $\left(N_{S}=5\right)$ and $\left(N_{S}=15\right)$. In the first case $\left(N_{S}=5\right)$, the estimation accuracy was $2.4(\mathrm{~Hz})$ for MLPF and $4.3(\mathrm{~Hz})$ for MVPF, as shown in Fig. 9. In the second case $\left(N_{s}=15\right)$, the resulting accuracy was $2.1(\mathrm{~Hz})$ for MLPF and $3.2(\mathrm{~Hz})$ for MVPF, as shown in Fig. 10.

Note: Estimation accuracy is related to the standard deviation of estimation errors, as given in (11), [29].

$$
\sigma_{E S}=\sqrt{\frac{1}{M-1} \sum_{i=1}^{M}\left(d_{i}-\mu\right)^{2}}
$$

where $\sigma_{E S}$ is the Estimation Accuracy of a studied parameter, $M$ is the number of observations, $d$ is the estimation error that has the equation: $\left(d_{i}=\right.$ true $_{\text {value }}$ - estimated value $\left.{ }_{i}\right)$, and $\mu$ is the mean of estimation errors.

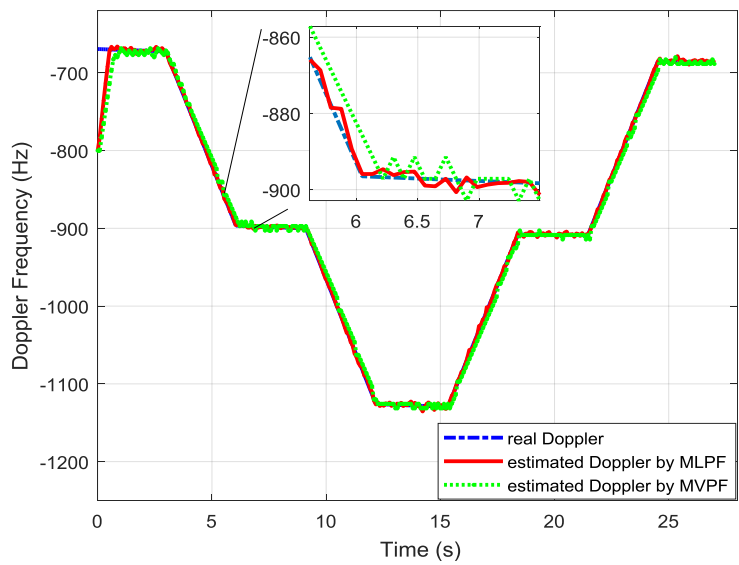

Fig. 9. Real and estimated Doppler frequency $\left(\mathrm{N}_{\mathrm{s}}=5\right)$

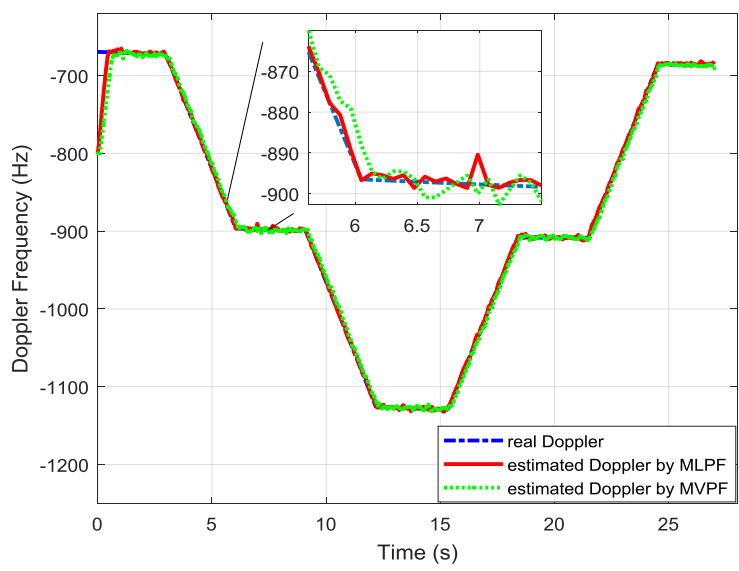

Fig. 10. Real and estimated Doppler frequency $\left(\mathrm{N}_{\mathrm{s}}=15\right)$

By focusing on Fig. 9 and Fig. 10, we notice the following points: First, MLPF and MVPF have convergent performance in terms of estimation accuracy, and their performance is improved by increasing the number of particles. Second MLPF has less complexity than MVPF because particles by MVPF are resampled based on calculating and analyzing the state probability of each particle, consequently the complexity of MVPF is increased by increasing the dimension of the state vector. Finally, these two types are suitable for tracking maneuvering targets, but the type (MVPF) has better performance for tracking these targets in the sudden environments, such as Decoy Flares or sudden crash of airplanes, because using this type leads to observe states that have low probabilities.

Note: The author of [2] had estimated the Doppler frequency of a maneuvering target by (MLPF) when he estimated target coordinates (at $\mathrm{SNR}=3 \mathrm{~dB}$ ) in the case of passive radar with multiple (DVB-T) transmitters and a receiver. The estimation accuracy in his case was approximately $2(\mathrm{~Hz})$, taking into consideration that the number of particles larger than our case. The lower value for the parameter (SNR) in [2] does not affect the comparison with the simulation results, because the particle filter can effectively estimate parameters that change very non-linearly at low SNR, [23], [24].

\section{CONCLUSION}

In this paper, the estimation of Doppler frequency of a maneuvering target has been performed by using the two types of particle filter: Maximum Likelihood Particle Filter and Minimum Variance Particle Filter. The characteristics of each type were checked by simulating a passive radar system that has the bistatic geometry (a DVB-T transmitter and a receiver). The simulation results have illustrated the efficiency of each type in terms of estimation accuracy, complexity, and a suitable application.

\section{REFERENCES}

[1] H. Kuschel, D. Cristallini and K. E. Olsen: Tutorial: Passive Radar Tutorial, IEEE Aerospace and Electronic Systems Magazine, vol.34, no.2, pp.2-19, 2019, DOI: 10.1109/MAES.2018.160146.

[2] K. Jeshy: "Tracking of maneuvering targets by passive radar using Gaussian Particle filter", Ph.D. dissertation, National Institute of Telecommunications, Pierre and Marie Curie University, Paris, France, 2012, (In French).

[3] W. Cao, X. Li, W. Hu, J. Lei, and W. Zhang: OFDM reference signal reconstruction exploiting subcarrier-grouping-based multi-level LloydMax algorithm in passive radar systems, IET Radar, Sonar \& Navigation, vol. 11, no. 5, pp. 873-879, 2017, DOI: 10.1049/ietrsn.2016.0340.

[4] L. Hongchao, L. Bing, G. Hongqi, and H. Biao: Reconstruction of reference signal for $D V B-S$ based passive radar systems, International Journal of Signal Processing Systems, vol.1, no. 1, pp. 116-120, 2013, DOI: 10.12720/ijsps.1.1.116-120.

[5] D. W. O'hagan, H. Kuschel, J. Heckenbach, M. Ummenhofer, and J. Schel: Signal reconstruction as an effective means of detecting targets in a DAB-based PBR, In 11-th International Radar Symposium, IEEE, 2010, pp. 1-4.

[6] M. Płotka, M. Malanowski, P. Samczyński, K. Kulpa, and K. Abratkiewicz: Passive Bistatic Radar Based on VHF DVB-T Signal, In 2020 IEEE International Radar Conference (RADAR), IEEE, 2020, pp. 596-600, DOI: 10.1109/radar42522.2020.9114859. 
[7] H.D. Griffiths: "PASSIVE BISTATIC RADAR AND WAVEFORM DIVERSITY", Defense Academy of the United Kingdom Shrivenham, United Kingdom, 2009.

[8] M. Conti, F. Berizzi, M. Martorella, E. Dalle Mese, D. Petri, and A. Capria: High Range Resolution Multi channel DVB-T Passive Radar, IEEE Aerospace and Electronic Systems Magazine, vol. 27, no. 10, pp. 37-42, October 2012.

[9] R. Zemmari, M. Broetje, G. Battistello and U. Nickel: GSM passive coherent location system: performance prediction and measurement evaluation, IET Radar, Sonar and Navigation, vol. 8, no. 2, pp. 94-105, 2014, DOI: 10.1049/iet-rsn.2013.0206.

[10] R. Tao, H. Z. Wu, and T. Shan: Direct-path suppression by spatial filtering in digital television terrestrial broadcasting-based passive radar, IET radar, sonar \& navigation, vol.4, no. 6, pp. 791-805, 2010, DOI: 10.1049/iet-rsn.2009.0138.

[11] B. Satar, G. Soysal, X. Jiang, M. Efe, and T. Kirubarajan: Robust Weighted $l_{1,2}$ Norm Filtering in Passive Radar Systems, Sensors, vol. 20, no. 11, 2020, DOI: 10.3390/s20113270.

[12] Y. Zhou, W. Xia, J. Zhou, L. Huang, and M. Huang: Coherent Integration Algorithm for Weak Maneuvering Target Detection in Passive Radar Using Digital TV Signals, In International Conference on Machine Learning and Intelligent Communications, Springer, Cham, 2017, pp. 215-224.

[13] M. Malanowski: Detection and parameter estimation of manoeuvring targets with passive bistatic radar, IET Radar, Sonar and Navigation, vol. 6, no. 8, pp.739-745, 2012, DOI: 10.1049/iet-rsn.2012.0072.

[14] G. Cui, J. Liu, H. Li, and B. Himed: Target detection for passive radar with noisy reference channel, In 2014 IEEE Radar Conference, IEEE, 2014, pp. 0144-0148. DOI:10.1109/radar.2014.6875573.

[15] X. Zhang, H. Li, J. Liu, and B. Himed: Joint delay and Doppler estimation for passive sensing with direct-path interference, IEEE Transactions on Signal Processing, vol.64, no. 3, pp. 630-640, 2016, DOI: 10.1109/TSP.2015.2488584.

[16] M. Malanowski, K. Kulpa, and J. Misiurewicz: Acceleration estimation for passive coherent location radar, In 2008 IEEE Radar Conference, IEEE, 2008, pp. 1-5, DOI: 10.1109/radar.2008.4721010.

[17] L. Zheng and X. Wang: Super-Resolution Delay-Doppler Estimation for OFDM Passive Radar, IEEE Transactions on Signal Processing, vol. 65, no. 9, pp. 2197-2210, 2017, DOI: 10.1109/TSP.2017.2659650.

[18] P. Krysik, M. Wielgo, J. Misiurewicz, and A. Kurowska: Doppler-only tracking in GSM-based passive radar, In 17th International Conference on Information Fusion (FUSION), IEEE, July 2014, pp. 1-7.

[19] A. Kazem, A. Malki, and A. M. Almanofi: Target Coordinates Estimation by a Passive Radar with a Single non-Cooperative Transmitter and a Single Receiver, Journal of Communications Software and Systems, vol. 16, no. 2, June 2020, DOI: 10.24138/jcomss.v16i2.984.

[20] N. Kovvali, M. Banavar, and A. Spanias: "An Introduction to Kalman Filtering with MATLAB Examples", SYNTHESIS LECTURES ON SIGNAL PROCESSING \#12, Morgan \& Claypool Publishers, 2014.

[21] P. S. Madhukar and L. B. Prasad: State Estimation using Extended Kalman Filter and Unscented Kalman Filter, In 2020 International Conference on Emerging Trends in Communication, Control and Computing (ICONC3), IEEE, 2020, pp. 1-4, DOI: 10.1109/iconc345789.2020.9117536.

[22] K. György, A. Kelemen, and L. Dávid: Unscented Kalman filters and Particle Filter methods for nonlinear state estimation, Procedia Technology, vol. 12, no. 1, pp. 65-74, 2014, DOI: 10.1016/j.protcy.2013.12.457.

[23] A. Kazem: "Generalized Deterministic Particles in non-Linear Filtering: Applications of Defense and communication", Ph.D. dissertation, LAAS Laboratory, France, 2008, (In French).

[24] A. Ziadi: "Deterministic Gaussian Particles in Non-Linear Maximum Likelihood: Application of optimal Filtering in Radar and GPS Signals", Ph.D. dissertation, LAAS Laboratory, France, 2007, (In French).

[25] K. Jishy and F. Lehmann: A Bayesian track-before-detect procedure for passive radars, EURASIP Journal on Advances in Signal Processing, no. 1, 2013, DOI: 10.1186/1687-6180-2013-45.

[26] C. Hue, J-P. Le Cadre, and P. Pérez: Tracking multiple objects with particle filtering, IEEE transactions on aerospace and electronic systems, vol. 38, no. 3, pp. 791-812, 2002, DOI: 10.1109/taes.2002.1039400.

[27] D. An, J.-H. Choi, and N. H. Kim: Prognostics 101: A tutorial for particle filter-based prognostics algorithm using Matlab, Reliability Engineering \& System Safety, vol. 115, pp.161-169, 2013, DOI: 10.1016/j.ress.2013.02.019.
[28] F. Gustafsson: Particle Filter Theory and Practice with Positioning Applications, IEEE Aerospace and Electronic Systems Magazine, vol. 25, no.7, pp.53-82, 2010, DOI: 10.1109/maes.2010.5546308.

[29] E.F. Bauermeister and D.W. O'Hagan: "On Particle Filters in Radar Target Tracking", Master thesis, Department of Electrical Engineering, University of Cape Town, August 2016.

[30] Y. Xu, K. Xu, J. Wan, Z. Xiong, and Y. Li: Research on particle filter tracking method based on Kalman filter, In 2018 2nd IEEE Advanced Information Management, Communicates, Electronic and Automation Control Conference (IMCEC), IEEE, 2018 pp. 1564-1568, DOI: 10.1109/imcec.2018.8469578.

[31] F. Lehmann: Deterministic particle filtering for GPS navigation in the presence of multipath, AEU-International Journal of Electronics and Communications, vol.63, no. 11, pp. 939-949, 2009, DOI: 10.1016/j.aeue.2008.07.009.

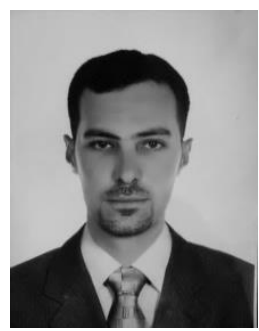

A. M. Almanofi was born in Damascus, Syrian Arab Republic in 1987. Received the Communication and Electronics engineering degree in 2011 from Damascus University. He received his Master degree in Communication from the Higher Institute for Applied Sciences and Technology (HIAST) in 2015. He is currently pursuing the Ph.D. degree in Passive Radars at (HIAST). His research interests are in localizing targets in Cartesian coordinates and estimating parameters of maneuvering targets by Particle Filter.

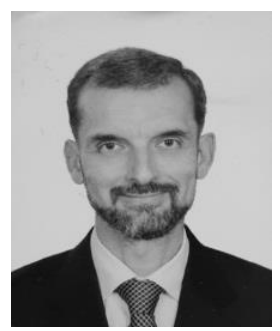

A. Malki was born in Damascus, Syrian Arab Republic in 1956. He received his Electrical Engineering degree (Honor) in 1979, from Damascus University. He received his DEA degree in Electronics in 1982, from the National Higher School of Aeronautics and Space, Toulouse, France. He received his Ph.D. degree in Electronics/ Microwave Circuit Design in 1985, from the National Higher School of Aeronautics and Space, Toulouse, France. During that period he worked with Thomson CSF, Space division at Toulouse, France. His main research interests include: design and development of a wide variety of microwave components and subsystems, such as filters, directional couplers, detectors, LNAs, medium and high power amplifiers, frequency synthesizers, $\mathrm{RF}$ and Microwave front ends. He is a senior professor at the Higher Institute for Applied Sciences and Technology (HIAST), Damascus, Syria.

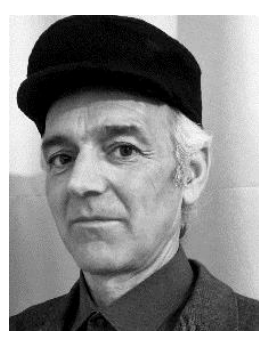

A. Kazem was born in Homs, Syrian Arab Republic in 1970. He received the Engineering and DES degrees in 1994, from the Higher Institute for Applied Sciences and Technology (HIAST), Damascus, Syria. He received his DEA degree in Automatics and Signal Processing in 2003, from the Laboratory for Analysis and Architecture of Systems (LAAS), Toulouse, France. In 2007, he received the Ph.D. degree in Random Signal Processing/Nonlinear Filtering from LAAS-CNRS. He worked as a research engineer with HIAST, Alcatel Alena Space-France, and DSI (Distribution Services Industrials), Toulouse, France. His research interests include statistical signal processing and its applications in radar, sonar, wireless communication systems, and identification systems. Since 2010, he has been a researcher in the Department of Electronic Engineering, HIAST, Damascus-Syria. 